\title{
Intratympanic methylprednisolone for sudden sensorineural hearing loss: comprehensive re examination of the model
}

\begin{abstract}
Objective: To evaluate the effectiveness and safety of Intratympanic methylprednisolone injection in patients with sudden sensorineural hearing loss (SSNHL) by means of a comparison between subjective and objective outcomes and a review of the literature regarding methylprednisolone transtympanic administration.
\end{abstract}

Data Sources: An electronic database search (MEDLINE and PubMed) was performed with the objective of identifying all studies published in the English language between January 1980 and October 2014 on the treatment of intratympanic methylprednisolone injection in ISSNHL. Prospective, non-randomized, case review of patients diagnosed with ISSNHL treated with intratympanic methylprednisolone from January 1, 2012 to June 1, 2014.

Study Selection: Twenty-seven articles describing intratympanic methylprednisolone injection in ISSNHL

Data Extraction: Mean, standard deviation and extreme values were presented in continuous variables; absolute value and proportions for categorical variables. The results were expressed as mean \pm standard deviation (SD). The comparison between categorical variables was evaluated by the $\mathrm{c} 2$ test or the continuity correction $\mathrm{c} 2$.

Data Synthesis: The entire analysis was performed using the SPSS package, version 15.0 (SPSS package Inc.)

Conclusion: Intratympanic methylprednisolone injection is a valid and safety option as a rescue therapy and also as first line treatment according to objective and subjective outcomes. It is the first time in which a correlation between subjective and objective outcomes could be established.

Keywords: inner ear, methylprednisolone, intratympanic therapy, sudden sensorineural hearing loss, steroids
Volume 3 Issue 2 - 2015

\author{
Jeanette Sáenz Piñones, Ithzel Villarreal, \\ Raimon García Chillerón, Rafael Ramírez- \\ Camacho , José Ramón García-Berrocal, Trini \\ dad \\ Department of Otorrinolaringology, Hospital Universitario \\ Puerta de Hierro Majadahonda University, Spain
}

Correspondence: Jeanette Sáenz Piñones, Department of Otorrinolaringology, Hospital Universitario Puerta de Hierro Majadahonda University, Spain, Tel 34645445472. Email jeanehesaenz@yahoo.com

Received: June 16,2015 | Published: September 18,2015
Abbreviations: SSNHL, sudden sensori neural hearing loss; ISSHL, idiopathic sudden sensori neural hearing loss; ITS, intratympanic steroids

\section{Introduction}

Since idiopathic sudden sensorineural hearing loss (ISSHL) is a true otologic emergency, a clear definition is mandatory. Although most authors agree that ISSHL can develop in 72hours, there is controversy in considering the defining intensity of the hearing loss. ${ }^{1-4}$ The natural history of ISSHL has not been elucidated; spontaneous recovery has been reported to occur in approximately $30-60 \%$ of $\operatorname{cases}^{5}$ and some cases recover so quickly that they do not seek medical attention.

The diagnosis of ISSHL is currently debatable due to the fact that the natural history of ISSHL is unknown and there is no single therapy that has been demonstrated to be effective. Due to ISSHL is a diagnosis of exclusion, the more extended and thorough the diagnostic investigation is conducted, the less idiopathic causes are found. Since the diagnostic work-up must exclude any identifiable causes, patients should be followed for at least 12 months, time enough to consider the diagnosis of ISSHL. ${ }^{6}$

Although the exact cause of ISSNHL is still controversial, the main theories include viral infection of the labyrinth, vascular insult and autoimmunity. The lack of consensus in the management of ISSNHL is due to difficulty in finding the real cause of the hearing loss. Most recoveries occur within the first two weeks after the onset. Recovery is mainly affected by the degree and type of hearing loss and time to therapy. Attempts to look for a consensus are recommended. ${ }^{7}$ If for refractory hearing loss the expected recovery is extremely low, for a first-line therapy a confounding factor is the presence of the spontaneous recovery. However, when a patient seeks for medical advice and attention, usually days or weeks after the onset of the hearing loss, we can hypothesize that spontaneous recovery of hearing is unlikely. Therefore, in such a situation, no treatment or the use of placebo may be questionable and raises ethical dilemmas.

Systemic steroid therapy is a common treatment modality, with a reported success rate between $5 \%$ and $89 \% .^{8-10}$

One of the main advantages of systemic steroids is their ability to arrest an immune reaction in the context of an autoimmune disorder. When the inner ear is the only organ affected by immunological responses, e.g. ISSHL with suspected immunologic origin, a clinical profile for the diagnosis is valuable and corticosteroids achieve the best outcomes in such as patients. ${ }^{11}$ Despite their widespread use, there is little consensus on the effectiveness of oral steroids and recent systematic reviews have called standard oral steroid therapy 
into question. ${ }^{12}$ For patients failing to recover after initial oral or intravenous steroids, there were no additional options.

Silverstein et al., ${ }^{13}$ first applied the Intratympanic steroid injection as treatment of ISSHL. Most of the studies regarding Intratympanic steroids (ITS) have shown their efficacy as an initial treatment for ISSNHL (as the first treatment without systemic corticosteroids), adjuvant therapy (concomitantly with systemic corticosteroids) or as a salvage therapy for the refractory ISSHL patients (started after systemic corticoid therapy has failed). The aims of the present study were the determination of the effectiveness and safety of Intratympanic methylprednisolone to treat ISSHL by means of a comparison between subjective and objective outcomes and a review of the literature regarding to trans tympanic intervention focused on methylprednisolone administration.

\section{Patients and methods}

Prospective, non-randomized, case review of patients diagnosed with ISSNHL treated with Intratympanicl methylprednisolone.

\section{Inclusion criteria}

a. SSNHL, which was defined as a sudden unilateral sensorineural hearing loss of at least $30 \mathrm{~dB}$ at 3 contiguous frequencies over a period of $\leq 3$ days.

b. Time from the onset of hearing loss to the treatment was $\leq 14$ days

c. No history of ear diseases.

d. No specific causes for the SSNHL after proper investigation.

e. Recovered less than $50 \%$ of their pre-loss hearing during systemic steroid treatment and presented for Intratympanic therapy within 1 month (up to 34days) of onset (rescue group) or subjects affected by systemic disorders that do not recommend systemic steroid therapy (first line group).

The exclusion criteria were defined as follows

a. Bilateral hearing loss

b. Other contraindications to the administration of Intratympanic (IT) steroids

c. The presence of a neoplasm or recent chemotherapy or radiation therapy

d. Congenital cochlear malformations or the presence of otitis media with an abnormal Tympanogram

e. Recent use of ototoxic medications

f. Liver or renal dysfunction, and/or pregnancy. Our local ethical committee granted approval for the study, provided that the patient made the choice of treatment. All the patients included in the study were informed about each treatment and selected their preferred type of therapy of their own free will.

\section{Rescue group}

Seventeen patients had failure of systemic therapy and received injections as a rescue line. Of them, three were treated with intravenous methylprednisolone $125-500 \mathrm{mg}$ for $72 \mathrm{hours}$, fourteen received oral steroids (methylprednisolone or deflazacor) $1 \mathrm{mg} / \mathrm{kg} /$ day tapered in three weeks and two were treated in other hospital and we could not get the records.

\section{First line group}

Four patients were treated with local steroids because of their medical records: one had a vascular necrosis of hip, one had schizophrenia and two had hypertension treated with several drugs. Subjects received 4 methylprednisolone injections through the tympanic membrane within a 1-week period. Most injections were administered in the outpatient department of otolaryngology by one of the authors (JR G-B).

\section{Intervention}

The patient was placed in a supine position with the affected ear angled toward the ceiling. The external ear canal was cleaned of debris and the tympanic membrane was visualized with an operating microscope. Local anaesthetic of topical $5 \%$ lidocaine warmed to body temperature solution filled the external canal and tympanic membrane. A 1-ml tuberculin syringe and a 27-gauge spinal needle slightly angled to allow a proper visualization of the puncture site were used to inject $0.3-0.5 \mathrm{ml}$ of $40 \mathrm{mg} / \mathrm{ml}$ methylprednisolone solution which penetrated the tympanic membrane at the posterior-inferior quadrant, avoiding puncturing in the same place twice during the course of the treatment. The injection was administered slowly so that the solution pooled around the round window niche, completely filling the middle ear cavity leaving any surplus fluid in the external auditory canal. The patient was instructed to avoid swallowing; speaking or moving in the supine position with the head tilted 45 degrees to the healthy side for 30 minutes to provide a maximal absorption of the medication through the round window and to prevent drug leakage through the eustaquian tube. The dose varied due to subject specific factors, although in most cases, at least $0.3 \mathrm{ml}$ was injected.

\section{Hearing evaluation}

Patients were evaluated with pure-tone audiometry before each injection and 1 month after steroid injection. The mean value for the pre treatment and post treatment was calculated as the mean value at each of the 8 frequencies $(125,250,500,1000,2000,4000$ and 8000 $\mathrm{Hz})$ and also at 5 frequencies $(250,500,1000,2000,4000)$.

\section{Standard Assessment}

The standard assessment included routine audiometric testing (PTA at five frequencies and impedance audiometry), serological tests (ANA, FTA, inmunophenotype of blood T cells), serum glucose measurement, coagulation and MRI of posterior fossa.

\section{Outcome assessment}

Hearing response was defined as an improved pure-tone average $\geq 10 \mathrm{~dB}$, Subjective outcome was considered when a statistical difference in a visual analogue scale (VAS) pre treatment and post treatment was observed. The visual analogue scale was used to describe hearing loss and tinnitus in a subjective way. It was expressed from 0 to 10 , indicating that 0 was no symptom and 10 the most annoying o incapacitating symptom.

\section{Statistical analysis}

Mean standard deviation and extreme values were presented in continuous variables; absolute value and proportions for categorical variables. The results were expressed as mean \pm standard deviation (SD). The comparison between categorical variables was evaluated by the $\mathrm{c} 2$ test or the continuity correction $\mathrm{c} 2$. The results for each frequency between pre and post comparisons were analysed using $t$-tests for paired data. The mean of the differences have been estimated by 
calculating their confidence intervals of $95 \%$. The Pearson correlation coefficient for the measures of relationship VAS and the global mean. The box plot was drawn to illustrate the median, quartiles and extreme values of VAS. The level of statistical significance was $p<0.05$ and two side. The entire analysis was performed using the SPSS package, version 15.0 (SPSS package Inc.)

\section{Results}

Twenty-one patients enrolled in the study. There were 12 males and 9 females. The mean age was 46 years and ranged from 27 to 85 years. Seventeen patients $(80.95 \%)$ had failure of systemic therapy and received injections as a rescue line. Of them, three were treated with intravenous methylprednisolone for 72 hours, fourteen received oral steroids (methylprednisolone or deflazacor) for 21days and two were treated in other hospital and we couldn't get the records. The mean days from the onset of systemic therapy to the injections were 11.5days. Four patients (19.04\%) had contraindications for systemic therapy: two with hypertension, one had a previous a vascular necrosis of the hip and one had schizophrenia. In these cases, the tympanic injection was their first-line treatment.

All patients received one weekly injection for 4 weeks. Eleven patients $(52.3 \%)$ did not express any complication. Seven patients $(33.3 \%)$ experienced pain that disappear in minutes, four patients
(19\%) described dizziness and one (4.8\%) had burning sensation. There was a case of perforation in a patient that had a monomer tympanic membrane. The mean value for hearing loss in the VAS before and after the treatment was 8.6 and 1.4, respectively. The mean of the difference in score was $7.1(5.1-9.2 ; p<0.001)$ (Figure 1).

The mean value for tinnitus in the VAS before and after the treatment was 7.8 and 2.9 , respectively. The mean of the difference in score was 4.9 (3.1- 6.6; $p<0.001)$.Of the 21 patients, $14(66.7 \%)$ presented a PTA average $>10 \mathrm{~dB}$ hearing thresholds at 250-500-1000$2000-4000 \mathrm{~Hz}$ before and after the treatment were $59.0 \mathrm{~dB}$ and 40.4 $\mathrm{dB}$, respectively and the improvement was $18.75 \mathrm{~dB}(\mathrm{p}<0.001)$. Table 1 represents each frequency $(125-8000 \mathrm{~Hz})$ with their means and standard deviation. We also used these frequencies to get the mean of hearing loss with the VAS and it was 7.4 before and 3.0 after, with a mean of $4.4(\mathrm{p}<0.001)$. The Pearson correlation between VAS (hearing loss $)$ and the PTA average pre treatment was $0.53(p=0.13)$ and post treatment was $0.67(p=0.01)$. We separate the total of patients in the two subgroups (first line and rescue) to evaluate if there is a significant difference in the results. In the first line group (4 patients) we got that the PTA pre treatment and post treatment using the five frequencies $(250-4000 \mathrm{~Hz})$ were $46.8 \mathrm{~dB}$ and $29.3 \mathrm{~dB}$ and the improvement was 17.5 ( $p=0.138)$. On the other hand, in the rescue group (17 patients), the PTA pre treatment and post treatment were $61.9 \mathrm{~dB}$ and $43.1 \mathrm{~dB}$ and the improvement was $18.8(p<0.01)$.

Table I Hearing thresholds before and after injections in each frequency

\begin{tabular}{|c|c|c|c|c|c|c|c|}
\hline Frequency & $\begin{array}{l}\text { Mean } \\
\text { Pretreatment(dB) }\end{array}$ & $\begin{array}{l}\text { SD pre } \\
\text { Treatment(dB) }\end{array}$ & $\begin{array}{l}\text { Mean post } \\
\text { Treatment(dB) }\end{array}$ & $\begin{array}{l}\text { SD post } \\
\text { Treatment(dB) }\end{array}$ & Improvement(dB) & $\mathrm{Cl} 95 \%$ & $\mathbf{p}$ \\
\hline 125 & 52.38 & 22.83 & 35.48 & 24.13 & 16.91 & $7.2-26.6$ & 0.002 \\
\hline 250 & 50.95 & 26.58 & 35.48 & 27.69 & 15.48 & I.3-29.7 & 0.034 \\
\hline 500 & 59.05 & 25.27 & 38.81 & 28.8 & 20.24 & $9.9-30.5$ & 0.001 \\
\hline 1000 & 59.05 & 23.27 & 40.95 & 25.72 & I8. I & $9.7-26.5$ & $<0.001$ \\
\hline 2000 & 56.67 & 19.06 & 40.24 & 24.87 & 16.43 & $6.9-25.8$ & 0.002 \\
\hline 4000 & 65 & 22.97 & 46.67 & 24.51 & 18.33 & $9.8-26.8$ & $<0.001$ \\
\hline 8000 & 68.25 & 25.66 & 49.75 & 28.77 & 18.5 & $9.5-27.4$ & $<0.001$ \\
\hline
\end{tabular}

$\mathrm{dB}$ : Decibeles; SD: Standard deviation

\section{Discussion}

ISSHL can be considered the result of abnormal activation of cellular stress pathway involving nuclear factor $\kappa \beta$ in supporting cells and spiral ligament fibrocystic resulting in the production of inflammatory cytokines and other stress-related proteins that can disrupt the homeostatic balance of the inner ear., ${ }^{6,14}$ Traditionally the effect of corticoids has been attributed to the anti-inflammatory and immunosuppressive activity including down-regulation of local proinflammatory cytokines (IL-1, IL- 6 , TNF- $\alpha$ ) ${ }^{15}$ neuroprotective, antioxidant and ant apoptotic effects, ion homeostasis (up regulation of aquaporins, increasing $\mathrm{Na}+-\mathrm{K}+$ exchange in the stair vascular is and direct effect on connexion-protein expression) and promotion of cochlear blood flow. Furthermore, glucocorticoids and mineral corticoid receptors have been demonstrated in the human inner ear with the highest concentration in the spiral ligament. ${ }^{16}$

Several trials showed that Intratympanic treatment was not inferior to oral steroid in hearing recovery. ${ }^{17-22}$ Although Intratympanic steroids for salvage have not yet been established as the gold standard for patients with incomplete recovery from ISSHL, mounting evidence for their effectiveness has been reported. ${ }^{23-25}$ Although dramatic recoveries in patients with profound ISSHL after salvage treatment with Intratympanic steroids are rare. The injections were offered to patients because we consider unethical not giving an alternative in patients with risk factors to systemic therapy. The Trans tympanic route has 2 advantages; firstly, it allows a greater concentration of drugs in the perilymph and secondly, it minimizes systemic effects and absorption. Side effects of systemic steroids can greatly compromise a person's quality of life and hinder their capacity to work throughout the treatment period. ${ }^{26}$

Based on Parnes et al report ${ }^{27}$ in which methylprednisolone presented the best absorption profile in both perilymph and endolymph after trans tympanic administration, we only use methylprednisolone for Intratympanic therapy and focused on this drug the present review. Trans tympanic corticosteroid treatment, however, has potential limitations, such as the presence of barriers and air bubbles in the round window niche ${ }^{28}$ and loss of drug down the Eustachian tube. Intratympanic steroid injection is an effective, safe, cheap and welltolerated office based-procedure for the treatment of ISSHL.

Injection procedures include Intratympanic injection using a needle puncture technique, Intratympanic injection after myringotomy, steroid administration through tympanostomy tubes and continuous delivery of steroids via a micro pump embedded in the round window niche (Table 2). Most authors achieve local anaesthesia of the external auditory canal and tympanic membrane by different methods (Table 
3 ) in order to make comfortable the procedure. In our experience the most safety and simple method of achieving local anaesthesia is by means of topical lidocaine $5 \%$ solution warmed to body temperature. Infiltration of the vascular strip could cause blister formation in the external auditory canal skin and diffusion of the anaesthetic to the middle ear cleft resulting in vertigo and transient facial palsy. Injection of a drug mixture consisting of methylprednisolone and lidocaine ${ }^{29}$ may interfere with the effect of the steroid, increases the potential risk of sudden vertigo due to lidocaine diffusion to the labyrinth, may be as painful as an IT injection without any previous local anaesthesia ${ }^{30}$ and adds another variable for the outcome evaluation. The reason for the pain reported in patients undergoing trans tympanic injection could be the $\mathrm{pH}$ of the steroid solution ${ }^{31}$ because of the volume needed, whereas with targeted delivery administration by using a round window membrane micro catheter, ${ }^{32}$ the lower volume is less likely to cause discomfort. Potential disadvantages of micro catheter include the risk and expense of general anaesthesia, operating room time, perforations of the tympanic membrane and price of the device.

Table 2 Comparison of administration delivery of local methylprednisolone

\begin{tabular}{|c|c|}
\hline Administration & Author \\
\hline \multirow[t]{17}{*}{ Injection } & William H Slaterry ${ }^{8}$ \\
\hline & Dallan I 54 \\
\hline & John Xenellis ${ }^{24}$ \\
\hline & Fitzgerald ${ }^{44}$ \\
\hline & Rahmi Kiliçç \\
\hline & Guillermo Plaza ${ }^{47}$ \\
\hline & Dallan I ${ }^{46}$ \\
\hline & Yang Chen29 \\
\hline & Igor Texeira ${ }^{48}$ \\
\hline & Necmi Arslan ${ }^{38}$ \\
\hline & Dallan I ${ }^{42}$ \\
\hline & Peng $\mathrm{Li}^{35}$ \\
\hline & Steven D Rauch ${ }^{17}$ \\
\hline & Yide Zhou 25 \\
\hline & Tomás Labatut ${ }^{34}$ \\
\hline & Onur Gundogan ${ }^{41}$ \\
\hline & Our study \\
\hline \multirow[t]{5}{*}{ Micropump } & Richard D Kopke 32 \\
\hline & Stefan Plontke ${ }^{37}$ \\
\hline & France Van Wijck $k^{45}$ \\
\hline & Wandong She ${ }^{49}$ \\
\hline & Sébastian Barriat ${ }^{43}$ \\
\hline \multirow[t]{3}{*}{ Tube } & Gerard J Gianoli² \\
\hline & Avik Banerjee 22 \\
\hline & Jürgen Lautermann ${ }^{36}$ \\
\hline
\end{tabular}

Concerns with steroid administration through a tympanostomy tube are related to the dose administered, in many cases, by the own patient, and the higher risk of perforation of the tympanic membrane. ${ }^{22,33}$ Needles of different diameters have been used for ITS injection. In our study we used a 27 -gauge spinal needle, what facilitates the collapse of the injection site when the needle is pulled out and keeping the fluid in the middle ear. ${ }^{34}$ The patient is placed supine with the treated ear upward for 20-30minutes in most studies. The amount injected in the middle ear in published papers ranges from 0.3 to $0.5 \mathrm{~mL}$, which is approximately the middle ear volume. In our study we used an approximate dose of $0.5 \mathrm{~mL}$ of $40 \mathrm{mg} / \mathrm{mL}$. The optimal dose, however, is debatable and it is very difficult to estimate taking into consideration that the amount of drug filling the middle ear cleft could be reduced by the mixture with local anaesthesia or sodium bicarbonate, the middle ear cleft size, the existence of adhesions in the round window niche and the loss through the eustaquian tube. ${ }^{35}$ Another concern are the exact treatment duration and schedule. Most protocols include from 1 to 4 injections with different time courses of administration (Table 4).

Table 3 Comparison of anesthesia in methylprednisolone local administration

\begin{tabular}{|c|c|}
\hline Anesthesia & Author \\
\hline $\begin{array}{l}\text { Alphacaine [ articaine hydrochloride } \\
40 \mathrm{mg} \text { epinephrine } 0006 \mathrm{mg} / \mathrm{ml} \text { ] }\end{array}$ & Sébastian Barriat ${ }^{43}$ \\
\hline EMLA & $\begin{array}{l}\text { Gerard J Gianoli }{ }^{2} \\
\text { Igor Texeira }{ }^{48}\end{array}$ \\
\hline General & Richard D Kopke ${ }^{32}$ \\
\hline Lidocaine & William H Slattery ${ }^{8}$ \\
\hline Lidocaine $1 \%$ + adrenaline I: 100000 & France Van Wijck ${ }^{45}$ \\
\hline injection & Tomás Labatut ${ }^{34}$ \\
\hline Lidocaine $10 \%$ [cotton ball soaked] & Onur Gundogan ${ }^{41}$ \\
\hline $\begin{array}{l}\text { Lidocaine hydrochloride } 2 \% \text { added in } \\
\text { the solution }\end{array}$ & John Xenellis ${ }^{24}$ \\
\hline \multirow[t]{2}{*}{ None } & Necmi Arslan ${ }^{38}$ \\
\hline & Rahmi Kiliç39 \\
\hline \multirow[t]{3}{*}{ Not specified } & I Dallan ${ }^{54}$ \\
\hline & I Dallan ${ }^{46}$ \\
\hline & Stefan Plontke ${ }^{37}$ \\
\hline \multirow[t]{8}{*}{ Phenol } & Gerard J Gianoli² \\
\hline & Avik Banerjee 22 \\
\hline & Fitzgerald ${ }^{44}$ \\
\hline & Guillermo Plaza ${ }^{47}$ \\
\hline & Peng $\mathrm{Li}^{35}$ \\
\hline & Steven D Rauch ${ }^{17}$ \\
\hline & Benjamin JWycherly ${ }^{50}$ \\
\hline & Yide Zhou ${ }^{25}$ \\
\hline \multirow[t]{2}{*}{ Tetracaine } & Yang Chen ${ }^{29}$ \\
\hline & Wandong She ${ }^{49}$ \\
\hline Topic lidocaine & Our study \\
\hline
\end{tabular}

Table 4 Comparison between intervals of methylprednisolone administration

\begin{tabular}{|c|c|}
\hline Interval & Author \\
\hline I alternating days injection [total 3] & Igor Texeira ${ }^{48}$ \\
\hline I alternating days injection [total 4] & Yide Zhou ${ }^{25}$ \\
\hline I alternating days injection [total 5] & Necmi Arslan ${ }^{38}$ \\
\hline I consecutive diary injection [total 5] & Jürgen Lautermann ${ }^{36}$ \\
\hline \multirow[t]{2}{*}{ I injection } & I Dallan ${ }^{54}$ \\
\hline & I Dallan ${ }^{46}$ \\
\hline I injection each 2 days [ total 4] & Yang Chen ${ }^{29}$ \\
\hline \multirow[t]{2}{*}{ I injection each 3 days [ total 4] } & Peng $\mathrm{Li}^{35}$ \\
\hline & Onur Gundogan ${ }^{41}$ \\
\hline I injection each 3 days [ total 5] & Rahmi Kiliç ${ }^{39}$ \\
\hline I injection or more & I Dallan ${ }^{42}$ \\
\hline \multirow[t]{3}{*}{ I weekly injection [ total 3] } & Fitzgerald ${ }^{44}$ \\
\hline & Guillermo Plaza ${ }^{47}$ \\
\hline & Benjamin J Wycherly ${ }^{50}$ \\
\hline I weekly injection [ total 4] & Our study \\
\hline 2 weekly injections until improvement & Avik Banerjee ${ }^{22}$ \\
\hline \multirow[t]{2}{*}{ Not specified } & France Van Wijck ${ }^{45}$ \\
\hline & Sébastian Barriat ${ }^{43}$ \\
\hline Perfusion 4 weeks & Stefan Plontke ${ }^{37}$ \\
\hline Perfusion for 10 days & Wandong She ${ }^{49}$ \\
\hline Perfusion for 14 days & Richard D Kopke ${ }^{32}$ \\
\hline \multirow[t]{2}{*}{ Separated injection in 10-14 days [total 4] } & Gerard J Gianoli \\
\hline & William H Slattery ${ }^{8}$ \\
\hline
\end{tabular}


In the present study we performed 4 injections with one-week interval. Perhaps due to the pharmacodynamics of corticosteroids in the inner ear, the IT injections might be done with at least 24-hour intervals, but the risks of overdose and perforation of the tympanic membrane advise a more prolonged interval. The reported effective rates ranges from 12 to $100 \%$ and the average PTA improvement ranged from 8 to $62 \mathrm{~dB} \cdot{ }^{36,37}$ Most authors include $0.5,1,2$, and $4 \mathrm{kHz}$. The most widely adopted criteria for hearing recovery is the improvement of $10 \mathrm{~dB}$ in PTA after treatment. ${ }^{38,39}$ Since this criterion can overestimate the effectiveness of steroids, we included all frequencies $(0.125$ to $8000 \mathrm{~Hz})$ for calculating hearing recovery. According to this criterion, $66.7 \%$ of our patients showed a positive response. However, we could not establish significantly differences between the rescue group and first line group of patients due to the low number of cases in the first line group. Efficacy of treatment was also categorized according to Siegel's criteria ${ }^{40,41}$ relative gain ${ }^{42}$ and the speech reception thresholds (SRT), speech discrimination testing (SDS) and the maximum speech intelligibility. ${ }^{43-45}$

Many authors agree that patients that started therapy soon after failures of systemic therapy was detected had an evident advantage. ${ }^{46,47}$ In the present study time elapsed from onset of the symptoms to the beginning of the ITS injections was 11.3days. This short period could represent a prognosis factor in the outcome of ISSHL and it was similar to observed by other authors. ${ }^{8,32,48,49}$ A significantly increased response rate was found in patients having an audiogram $>5$ weeks after the first dose of ITS. ${ }^{50}$ In our protocol we performed the last audiogram for the outcome assessment 4weeks after the last ITS administration. This follow-up would identify early versus late responders in base to different mechanisms of damaging the inner ear.

Although the possible ototoxic effect of IT steroids has been ruled out in experimental and clinical studies ${ }^{51-53}$ one patient with worsening of the PTA with repeated injections of methylprednisolone has been reported. ${ }^{54}$ This worsening may represent a dose-effect response: a higher than therapeutic dose might have damaged the inner ear. In our study, no patient experienced worsening of their hearing. Although IT steroid procedure was well tolerated, in our study seven patients (33.3\%) experienced pain that spontaneously disappearing in minutes or easily controlled by the administration of paracetamol. A recent report that compared the pain level of local anaesthesia, showed no difference in pain intensity among three methods (application of EMLA cream, subcutaneous injection of lidocaine 1\% with 1:100.000 epinephrine and IT injection without previous anaesthesia). A burning sensation around the ear was one of the most frequently encountered side effects of IT methylprednisolone, and it resolved over a period of 10-20minutes after the injection in most patients. ${ }^{55}$ In Rauch et al study (17), $54 \%$ of patients experienced pain.

Effective drug delivery into the inner ear can stimulate the vestibule and induce dizziness, possibly due to caloric effect, which implies a good prognosis. ${ }^{29}$ Four patients (19\%) complained of vertigo (despite of the solution was warmed to body temperature) or an increase in tinnitus during the injections, but these complaints resolved within minutes. Tympanic membrane perforations are usually observed if myringotomy had been done, and usually recover easily. ${ }^{32}$ In our study only one patient developed a tympanic membrane perforation. The use of a needle of 27-gauge that causes a small hole and the fact that injections were repeated at one-week intervals could facilitate the healing of the tympanic membrane thus justifying the low percentage of perforations found in our patients.

We propose the addition of a VAS (Visual Analogue Scale) to contribute to the evolution assessment of hearing recovery and tinnitus amelioration in ISSHL, based in a preliminary report presented in $2013 .^{56} \mathrm{~A}$ statistically difference in the VAS before and after the treatment in tinnitus and hearing loss could be observed $(p<0.001)$. Correlation between VAS (hearing loss) and the PTA average pretreatment and post treatment was statistically significant as well ( $p=$ $0.01)$. This is the first time, in our knowledge, that such a correlation has been reported.

\section{Conclusion}

ISSHL remains a challenging clinical problem. ISSHL can be considered the result of abnormal activation of cellular stress pathway inside the inner ear resulting in the production of inflammatory cytokines and other stress-related proteins that can disrupt the homeostatic balance of the inner ear. The main mechanism of action of steroids on cochlear function has been attributed to the antiinflammatory and immunosuppressive activity. Tran's tympanic route allows a greater concentration of drugs in the perilymph minimizing systemic adverse effects. Since methylprednisolone presented the best absorption profile we used this drug for treating patients affected by ISSHL.

Intratympanic steroid injection is an effective, safe, cheap and well-tolerated office based-procedure for the treatment of ISSHL. The low rate of complications and the hearing outcomes support its use not only as a rescue therapy but also as first line treatment. Subjective outcomes regarding to hearing loss and tinnitus strongly advocate the use of Intratympanic injection of methylprednisolone in ISSHL. It has to be considered that the second group has a small number of patients to extract definitive conclusions despite the results and that a study with a big number is needed to confirm the results.

\section{Acknowledgments}

None.

\section{Conflicts of interest}

Author declareas there are no conflicts of interest.

\section{Funding}

None.

\section{References}

1. Hughes GB, Freedman MA, Haberkamp TJ, et al. Sudden sensorineural hearing loss. Otolaryngol Clin North Am. 1996;29:393-405.

2. Gianoli GJ, Li JC. Transtympanic steroids for treatment of sudden hearing loss. Otolaryngol Head Neck Surg. 2001;125(3):142-146.

3. Battaglia A, Burchette R, Cueva R. Combination therapy (intratympanic dexamethasone + high-dose prednisone taper) for the treatment of idiopathic sudden sensorineural hearing loss). Otol Neurotol. 2008;29(4):453-460.

4. Haberkamp TJ, Tanyeri HM. Management of idiopathic sudden sensorineural hearing loss. Am J Otol. 1999;20(5):587-595.

5. Byl FM Jr. Sudden hearing loss: eight years experience and suggested pronostic table. Laryngoscope. 1984;94(5 Pt 1):647-661.

6. García-Berrocal JR, Ramírez-Camacho R, et al. Sudden presentation of immune-mediated inner ear disease: characterization and acceptance of a cochleovestibular dysfunction.J Laryngol Otol. 2003;117(10):775-779.

7. Plaza G, Durio E, Rivera T, et al.Consenso sobre diagnóstico y tratamiento de la sordera súbita. Acta Otorrinolaringol Esp. 2011; 62(2):144-157. 
8. Slattery WH, Fisher LM, Iqbal Z, et al.Intratympanic steroid injection for treatment of idiopathic sudden hearing loss. Otolaryngol Head Neck Surg. 2005;133(2):251-259

9. Chandrasekhar SS. Intratympanic dexamethasone for sudden sensorineural hearing loss: clinical and laboratory evaluation. Otol Neurotol. 2001;22(1):18-23.

10. Zadeh MH, Storper IS, Spitzer JB. Diagnosis and treatment of suddenonset sensorineural hearing loss: a study of 51 patients. Otolaryngol Head Neck Surg. 2003;128(1):92-98.

11. García Berrocal JR, Ramírez-Camacho R. Sudden sensorineural hearing loss: supporting the immunologic theory. Ann Otol Rhinol Laryngol. 2002;111(11):989-997.

12. Wei BPC, Muribu S, O'Leary S. Steroids for idiopathic sudden sensorineural hearing loss. Cochrane Database Syst Rev. 2006;7.

13. Silverstein $\mathrm{H}$, Choo $\mathrm{D}$, Rosenberg SI, et al. Intratympanic steroid treatment of inner ear disease and tinnitus (preliminary report). Ear Nose Throat J. 1996;75(8):468-471.

14. Ramírez-Camacho R, García-Berrocal JR, Trinidad A, et al. Centra role of supporting cells in cochlear homeostasis and pathology. Med Hypothesis. 2006;67(3):550-555.

15. García-Berrocal JR, Ramírez-Camacho R, Trinidad A, et al.Glucocorticoids: the best therapy for immune-mediated inner ear disease. Curr Topics Steroid Res. 2004;4:99-104.

16. Rarey KE, Curtis LM. Receptors for glucocorticoids in the inner ear. Otolaryngol Head Neck Surg. 1996;115(1):38-41.

17. Rauch SD, Halpin CF, Antonelli PJ, et al. Oral vs intratympanic corticosteroid therapy for idiopathic sudden sensorineural hearing loss: a randomized trial. JAMA. 2011;305(20):2071-2079.

18. Kakehata S, Sasaki A, Oji K, et al. Comparison of intratympanic and intravenous dexamethasone treatment on sudden sensorineural hearing loss with diabetes. Otol Neurotol. 2006;27(5):604-608.

19. Filipo R, Covelli E, Balsamo G, et al.Intratympanic prednisolone therapy for sudden sensorineural hearing loss: a new protocol. Acta Otolaryngol. 2010;130(11):1209-1213.

20. Dispenza F, Amodio E, De Stefano A, et al. Treatment of sudden sensorineural hearing loss with transtympanic injection of steroids as single therapy: a randomized clinical study. Eur Arch Otorhinolaryngol. 2011;268(9):1273-1278.

21. Hong SM, Park $\mathrm{CH}$, Lee JH. Hearing outcomes of daily intratympanic dexamethasone alone as a primary treatment modality for ISSHL. Otolaryngol Head Neck Surg. 2009;141(5):579-583.

22. Banerjee A, Parnes LS. Intratympanic corticosteroids for sudden idiopathic sensorineural hearing loss. Otol Neurotol 2005;26(5):878-881.

23. Spear SA, Schwartz SR. Intratympanic Steroids for Sudden Sensorineural Hearing Loss: A Systematic Review. Otolaryngol Head Neck Surg. 2011;145(4):534-543.

24. Xenellis J, Papadimitriou N, Nikolopoulos T, et al. Intratympanic steroid treatment in idiopathic sudden sensorineural hearing loss: A control study. Otolaryngol Head Neck Surg. 2006; 134(6):940-945.

25. Zhou Y, Zheng H, Zhang Q, et al.Early transtympanic steroid injection in patients with poor prognosis idiopathic sensorineural sudden hearing loss. ORL. 2011;73(1):31-37.

26. García-Berrocal JR, Ramírez-Camacho R, Lobo D, et al.Adverse effects of glucocorticosteroids therapy for inner ear disorders. ORL J Otorhinolaryngol Relat Spec. 2008;70(4):271-274.

27. Parnes LS, Sun AH, Freeman DJ. Corticosteroid pharmacokinetics in the inner ear fluids: an animal study followed by clinical application. Laryngoscope. 1999;109(7pt 2):1-17.
28. Alzamil KS, Linthicum FH Jr. Extraneous round window membranes and plugs: possible effect on intratympanic therapy. Ann Otol Rhinol Laryngol. 2000;109(1):30-32.

29. Chen Y, Wen L, Hu P, et al. Endoscopic Intratympanic Methylprednisolone Injection for Treatment of Refractory sudden sensorineural hearing loss and one case in pregnancy. J Otolaryngol Head Neck Surg. 2010;39(6):640-645.

30. Belhassen S, Saliba I. Pain assessment of the intratympanic injections: a prospective comparative study. Eur Arch Otorhinolaryngol. 2012;269(12):2467-2473.

31. Fetterman BL, Saunders JE, Luxford WM. Prognosis and treatment of sudden sensorineural hearing loss. Am J Otol. 1996;17(4):529-536.

32. Kopke RD, Hoffer ME, Wester D, et al.Targeted topical steroid therapy in sudden sensorineural hearing loss. Otol Neurotol. 2001;22(4):475-479.

33. Robey AB, Morrow T, Moore GF. Systemic side effects of transtympanic steroids. Laryngoscope. 2010;120(Suppl 4):S217.

34. Labatut T, Daza MJ, Alonso A. Intratympanic steroids as primary initial treatment of idiopathic sudden sensorineural hearing loss. The Hospital Universitario Ramón y Cajal experience and review of the literature. Eur Arch Otorhinolaryngol. 2013;270(11):2823-2832.

35. Li P, Zeng XL, Ye J, et al. Intratympanic Methylprednisolone Improves Hearing Function in Refractory Sudden Sensorineural Hearing Loss: A Control Study. Audiol Neurotol. 2011;16(3):198-202.

36. Lautermann J, Sudhoff H, Junker R. Transtympanic corticoid therapy for acute profound hearing loss. Eur Arch Otorhinolaryngol. 2005; 262(7):587-591.

37. Plontke S, Löwenheim H, Preyer S, et al. Outcomes research analysis of continuous intratympanic glucocorticoid delivery in patients with acute severe to profound hearing loss: Basis for planning randomized controlled trials. Acta Oto-Laryngologica. 2005;125(8):830-839.

38. Arslan N, Oğuz H, Demirci M, et al. Combined intratympanic and systemic use f steroids for idiopathic sudden sensorineural hearing loss. Otol Neurotol. 2011;32(3):393-397.

39. Kiliç R, Şafak MA, Oğuz H, et al. Intratympanic methylprednisolone for sudden sensorineural hearing loss. Otol Neurotol. 2007;28(3):312-316.

40. Siegel LG. The treatment of idiopathic sudden sensorineural hearing loss. Otolaryngol Clin North Am. 1975;8:467-473.

41. Gundogan O, Pinar E, Imre A, et al. Therapeutic Efficacy of the Combination of Intratympanic Methylprednisolone and Oral Steroid for Idiopathic Sudden Deafness. Otol Neurotol. 2013; 149(5):753-758.

42. Dallan I, Fortunato S, Casani AP, et al. Intratympanic methylprednisolone as first-line therapy in sudden sensorineural hearing loss: preliminary results from a case-control series. J Laryngol Otol. 2011; 125(10): 1004-1008.

43. Barriat S, van Wijck F, Staecker H, et al.Intratympanic Steroid Therapy Using the Silverstein Microwick TM for Refractory Sudden Sensorineural Hearing Loss Increases Speech Intelligibility. Audiol Neurootol. 2012;17(2):105-111.

44. Fitzgerald DC, McGuire JF. Intratympanic steroids for idiopathic sudden sensorineural hearing loss. Ann Otol Rhinol Laryngol. 2007;116(4):253-256

45. Van Wijck F, Staecker H, Lefebvre PP. Topical steroid therapy using the Silverstein MicrowickTM in sudden sensorineural hearing loss after failure of conventional treatment. Acta Oto-Laryngologica. 2007;127(10):1012-1017.

46. Dallan I, De Vito A, Fattori B, et al. Intratympanic methylprednisolone in refractory sudden hearing loss: A 27 patient case series with univariate and multivariate analysis. Otol Neurotol. 2009;31(1):25-30. 
47. Plaza G, Herráiz C. Intratympanic steroids for treatment of sudden hearing loss after failure of intravenois therapy. Otolaryngol Head Neck Surg . 2007;137(1):74-78.

48. Raymundo IT, Bahmad Jr F, Filho JB, et al. Intratympanic Methylprednisolone as Rescue Therapy in Sudden Sensorineural Hearing Loss. Braz J Otorhinolaryngol. 2010;76(4):499-509.

49. She W, Dai Y, Du X, et al. Hearing evaluation of intratympanic methylprednisolone perfusion for refractory sudden sensorineural hearing loss. Otolaryngol Head Neck Surg. 2010;142(2):266-271.

50. Wycherly BJ, Thompkins JJ, Kim HJ. Early Posttreatment Audiometry Underestimates Hearing Recovery after Intratympanic Steroid Treatment of Sudden Sensorineural Hearing Loss. Int J Otolaryngol. 2011.

51. Shirwany NA, Seidman MD, Tang W. Effects of transtympanic injection of steroids on cochlear blood flow, auditory sensitivity and histology in the guinea pig. Am J Otol. 1998;19(2):230-235.
52. Chandrasekhar SS, Rubinstein RY, Kwartler JA, et al. Dexamethasone pharmacokinetics in the inner ear: Comparison of route of administration and use of facilitating agents. Otolaryngol Head Neck Surg. 2000;122(4):521-528.

53. Yilmaz I, Yilmazer C, Erkan AN, et al.Intratympanic dexamethasone injection effects on transient-evoked otoacoustic emission. Am J Otolaryngol. 2005;26(2):113-117.

54. Dallan I, Bruschini L, Nacci A, et al. Transtympanic steroids as a salvage therapy in sudden hearing loss: preliminary results.ORL. 2006;68(5):185-190.

55. Topak M, Sahin-Yilmaz A, Ozdoganoglu T, et al. Intratympanic methylprednisolone injection for subjective tinnitus. J Laryngol Otol . 2009;123(11):1221-1225.

56. Sáenz-Piñones J, García-Berrocal JR, Villarreal $I$, et al. Methylprednisolone injection to the inner ear: an alternative to the systemic therapy. Paper presented at 50th Inner Ear Biology Workshop. Madrid, Spain. 2013. 